\title{
Novel Scheme for Minimal Iterative PSO Algorithm for Extending Network Lifetime of Wireless Sensor Network
}

\author{
Hemavathi $\mathbf{P}^{1}$, Nandakumar A. N. ${ }^{2}$ \\ ${ }^{1}$ Jain University, Bangalore, India \\ ${ }^{2}$ Dept of Computer Science and Engineering, New Horizon College of Engineering, Bangalore, India
}

\begin{tabular}{l} 
Article Info \\
\hline Article history: \\
Received Aug 17, 2017 \\
Revised Dec 28, 2018 \\
Accepted Jan 10, 2018 \\
\hline
\end{tabular}

Keyword:

Clustering

Energy

Power

PSO

Wireless sensor network

\begin{abstract}
Clustering is one of the operations in the wireless sensor network that offers both streamlined data routing services as well as energy efficiency. In this viewpoint, Particle Swarm Optimization (PSO) has already proved its effectiveness in enhancing clustering operation, energy efficiency, etc. However, PSO also suffers from a higher degree of iteration and computational complexity when it comes to solving complex problems, e.g., allocating transmittance energy to the cluster head in a dynamic network. Therefore, we present a novel, simple, and yet a cost-effective method that performs enhancement of the conventional PSO approach for minimizing the iterative steps and maximizing the probability of selecting a better clustered. A significant research contribution of the proposed system is its assurance towards minimizing the transmittance energy as well as receiving energy of a cluster head. The study outcome proved proposed a system to be better than conventional system in the form of energy efficiency.
\end{abstract}

Copyright () 2018 Institute of Advanced Engineering and Science. All rights reserved.

\section{Corresponding Author:}

Hemavathi P,

Jain University,

Bangalore, India.

Email: hemavathi.research@gmail.com

\section{INTRODUCTION}

There has been extensive research work carried out towards wireless sensor network for solving various types of issues e.g. energy issues [1], traffic management issues [2], security issues [3], routing issues [4], localization issues [5] etc. There has been a voluminous amount of investigation carried out in this area for addressing such issues, but still majority of the issues are yet to meet its full-proof solution. The primary cause of this problem is basically the sensor node, which is very small in size, posses low computational capabilities, and powered by a battery that has limited lifetime. The biggest challenge for the researchers to prove the applicability of their presented system in real-time sensor node that is not found to be discussed in majority of the existing research work. However, some of the researchers avoid such problems by considering either benchmark test bed, or adopt the configuration of some real-time motes e.g. Berkley Mote, MicaZ mote [6], etc. Hence, optimization is the best possibility in such scenario of node resources constraints. There are various methods by which optimizations have been carried out towards improving the performance of sensor node e.g. neural network, genetic algorithm, swarm intelligences etc [7]. Particle Swarm Optimization (PSO) is one such technique that uses many numbers of iterations in order to explore the best solution against the problems posed [8], [9]. From computational viewpoint, PSO enhances the candidate solution in the perspective of the anticipated outcomes and given problem in wireless sensor network. The problem is optimized by considering the candidate solution and its population (also called as particles) and this form of the candidate solution is subjected to iterative processing in order to obtain personal and global best outcome from the position and velocity of the particles. The primary reason behind the adoption of PSO is basically its intelligence-based approach that can be possibly implemented on any of 
problem (be it engineering or scientific). PSO is completely independent of computing mutation which has no overlapping. The velocity of the particle can be mechanism for initiating the search process and this makes the calculation process of PSO very simple. It also offers potentially better results as compared to other optimization techniques ever used in wireless sensor network. However, there is also certain associated usage of PSO-based mechanism in wireless sensor network. PSO is associated with partial-optimization problem that also tend to minimize the accuracy level of velocity factor of the particle as well as its direction. It is also not a preferred technique for solving the scattering problems in any wireless network. Most importantly, it is an iterative process and it will be required to store lots of information pertaining to its intermediate passes in order to perform comparative analysis of the elite outcome with respect to the personal and global best solution. However, there are various work carried out in existing system where the problems associated with the energy and clustering in wireless sensor network has been found solved by PSO-based approaches. In real sense, there are only few works in existing system which has amended original PSO implementation and hence, it is quite challenging to explore the level of effectiveness of existing system. Therefore, the proposed paper introduced a novel and simple attempt where the PSO is amended to get itself free from number of increasing iterative steps as a part of research contribution. The study outcome shows that proposed revised version of PSO offers better beneficial characteristics to both energy efficiency as well as data delivery system in wireless sensor network. Section 1.1 discusses about the existing literatures where different techniques are discussed for PSO based schemes used in solving multiple ranges of problems followed by discussion of research problems in Section 1.2 and proposed solution in 1.3. Section 2 discusses about algorithm implementation followed by discussion of result analysis in Section 3 . Finally, the conclusive remarks are provided in Section 4.

\subsection{Background}

Our prior research work has discussed different forms of approaches for retaining maximum energy in wireless sensor network [10]. This part of the study will further add different forms of contributions made by PSO in the area of wireless sensor network. Clustering-based approach was adopted for enhancing network lifetime using PSO as seen in the work of Zhou et al. [11]. Study towards cluster head selection is also carried out by $\mathrm{Ni}$ et al. [12] where PSO is used along with Fuzzy logic for optimizing clustering performance in sensor network. PSO algorithm was also found to optimize energy efficiency exclusively for software-defined aspects in sensory application. Xiang et al. [13] have presented a technique for energy conservation for software-defined sensor network. Issues of maintaining higher degree of fault tolerance while scheduling the allocation process of task can be also handled by PSO as seen in the work carried out by Guo et al. [14]. The work carried out by Parvin [15] has used PSO for overcoming non-participation process of nodes during aggregation process. Wu and Lin [16] have investigated the effect of PSO for exploring the specific absorption rate of wireless body area network. Study towards allocation of task is also carried out by Yang et al. [17] where the focus was laid on formulating transfer function and usage of mutation. Rahman and Matin [18] have presented their contribution towards enhancing the network lifetime using PSO for exploring the better position of the base station. Ho et al. [19] have used PSO for assisting in routing process for unmanned aerial vehicle using cooperative relay. Usage of PSO was seen in the work of Loscri et al. [20] who have used consensus aspects for searching better area in sensor network field. Chen et al. [21] have introduced a mechanism of charging deployment in order to enhance the optimality of energy performance in sensor network. Du et al. [22] have presented a mechanism of eliminating the electromagnetic interference while performing beaconing in wireless sensor network using PSO. The work carried out by Chen et al. [23] has used PSO as well as Cuckoo search technique in order to strengthen the security system of WSN. PSO was also used for addressing the self-localization problem in wireless sensor network by modifying some of its functionalities as seen in the work of Kun and Zhong [24]. Thilagavathi and Geetha [25] have presented a search algorithm for enhancing the residual energy of WSN. Elhabyan et al. [26] have presented a technique for enhancing the clustering operation while Huynh et al. [27] have developed a non-conventional PSO algorithm for prolonging the network lifetime taking case study of heterogeneous sensor network. Implementation of binary PSO for assisting in localization problem was seen in the work of Zain and Shin [28]. Cao et al. [29] have investigated the effectiveness of PSO by comparing with the conventional approach for solving localization problem. Jing et al. [30] have presented a similar approach where PSO was found to enhance the clustering operation of WSN. The combined work of Riaz and Srirammanoj [31] presented the sufficient authentication mechanism and achieved significant power redundancy in WSN lifetime. A novel review work on PSO based clustering routing protocol in WSN was found in Sun et al. [32] and hints for performance enhancement in the routing protocol. Rui et al. [33] discussed the clustering routing protocol in WSN and compared its performance with existing LEECH algorithm. This protocol provides the nodes energy balance and improves the network lifetime. Therefore, there are various variants of the PSO based

Novel Scheme for Minimal Iterative PSO Algorithm for Extending Network Lifetime of .... (Hemavathi P) 
approaches mainly to solve clustering, energy efficiency, and localization issues in wireless sensor network. The next section highlights the research problems that has been identified from the above mentioned research approaches.

\subsection{Problem Identification}

The problem identification of the proposed study is as follows:

a. Existing approaches using PSO has the split emphasis on clustering, energy efficiency, and localization problems where energy has not yet received a full proof solution.

b. The extent of amendments towards using the new version of PSO is very less and doesn't turn out to be a practically viable solution in large scale and dense networks.

c. Existing PSO techniques also renders a higher number of iterations to obtain better convergence performance. Therefore, it leads to computational complexity.

d. Compliance with standard energy modeling is less found to be adopted in existing literature, which without benchmarking is very hard to find its effectiveness against energy effectiveness.

Therefore, the problem statement of the proposed study can be stated as "Designing a computational friendly approach for formulating a novel selection of clusterhead to offer energy efficiency in a wireless sensor network."

\subsection{Proposed Solution}

The proposed system adopts an analytical research methodology to implement the optimization algorithm by enhancing the operation carried out by conventional PSO. Figure 1 highlight the design flow signifying that optimization of PSO was carried out by considering decision variables formulated by sensor nodes and their respective probability of becoming a clusterhead. The decision variables are also dependent on its size and bound (lower/higher) for effective control over the PSO iterations.

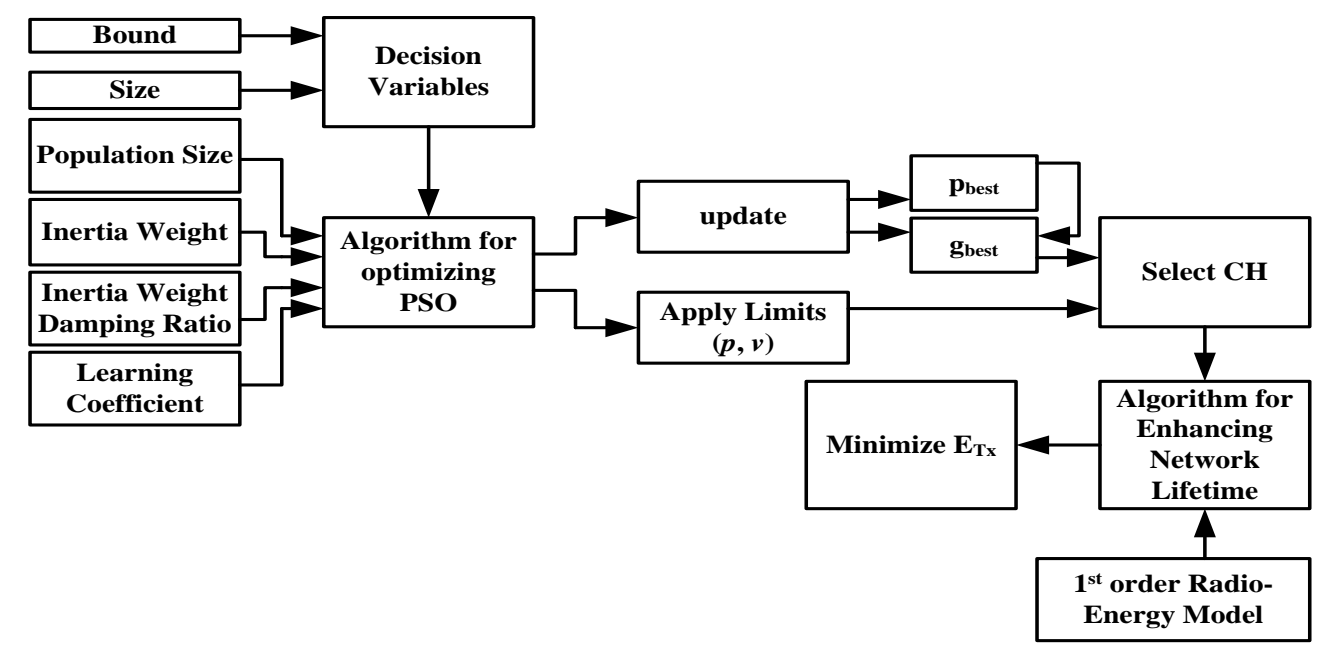

Figure 1. Design Flow of Proposed System

The contribution of the proposed system is its novelty introduced in PSO algorithm. The algorithm uses inertial weight, damping ratio, and learning coefficient (both local and global) to initially perform updating using a novel empirical approach to obtain personal best and global best. Using the limits applied to position and velocity along with global best outcome is used for selecting the effective clusterheads. An algorithm for enhancing network lifetime is designed using $1^{\text {st }}$ order Radio-Energy model for computing energy required to transmit and receive the data packet. Implementation of this model only ensures that proposed system adheres to an empirical methodology where energy modeling is formulated by its realdemands of communication. This operation is followed by minimizing the energy required for transmittance for the clusterhead that results in significant retention of the residual energy for a long run of the sensory application. The complete operation of saving the transmittance energy is carried out in two phases where the first phase focuses on the node to clusterhead and communication among clusterheads while the second phase focuses on only clusterheads to base station. The specific agenda of the proposed system is to minimize 
the iteration required to explore the global best solution in PSO that assists in minimizing overheads and any form of bottleneck condition owing to increasing traffic condition in a wireless sensor network. The next section discusses the algorithm implemented to pursue the flow of proposed research objectives as shown in Figure 1.

\section{ALGORITHM IMPLEMENTATION}

The proposed system offers an algorithm that is constructed by enhancing the PSO algorithm ONtwo purposes. The first purpose serves for optimizing the PSO performance by obtaining a global best solution with extremely less iterative step unlike conventional PSO and the second purpose is mainly to extend the residual energy of the sensor node as far as possible. The discussions of the algorithm are as follows:

\subsection{Algorithm for Optimizing PSO}

This algorithm is mainly responsible for ensuring that an effective cluster head is selected for offering better optimization performance with energy efficiency. It is believed that if the cluster head is selected properly than network lifetime could be positively enhanced. Here the selection is completely based on multiple parameters, e.g., number of nodes, the position of particles, velocity of particles, updating process, cost function involved in PSO methodology. The algorithm for optimizing the PSO is mainly carried out for reducing the number of iterative steps involved in exploring the global best function in PSO. For this purpose, the algorithm considers the input of $\eta$ (number of population), $p$ (position), $v$ (velocity), $\sigma$ (variance), and $r_{\max }$ (maximum rounds) that after processing leads to the result of sol best (best solution).

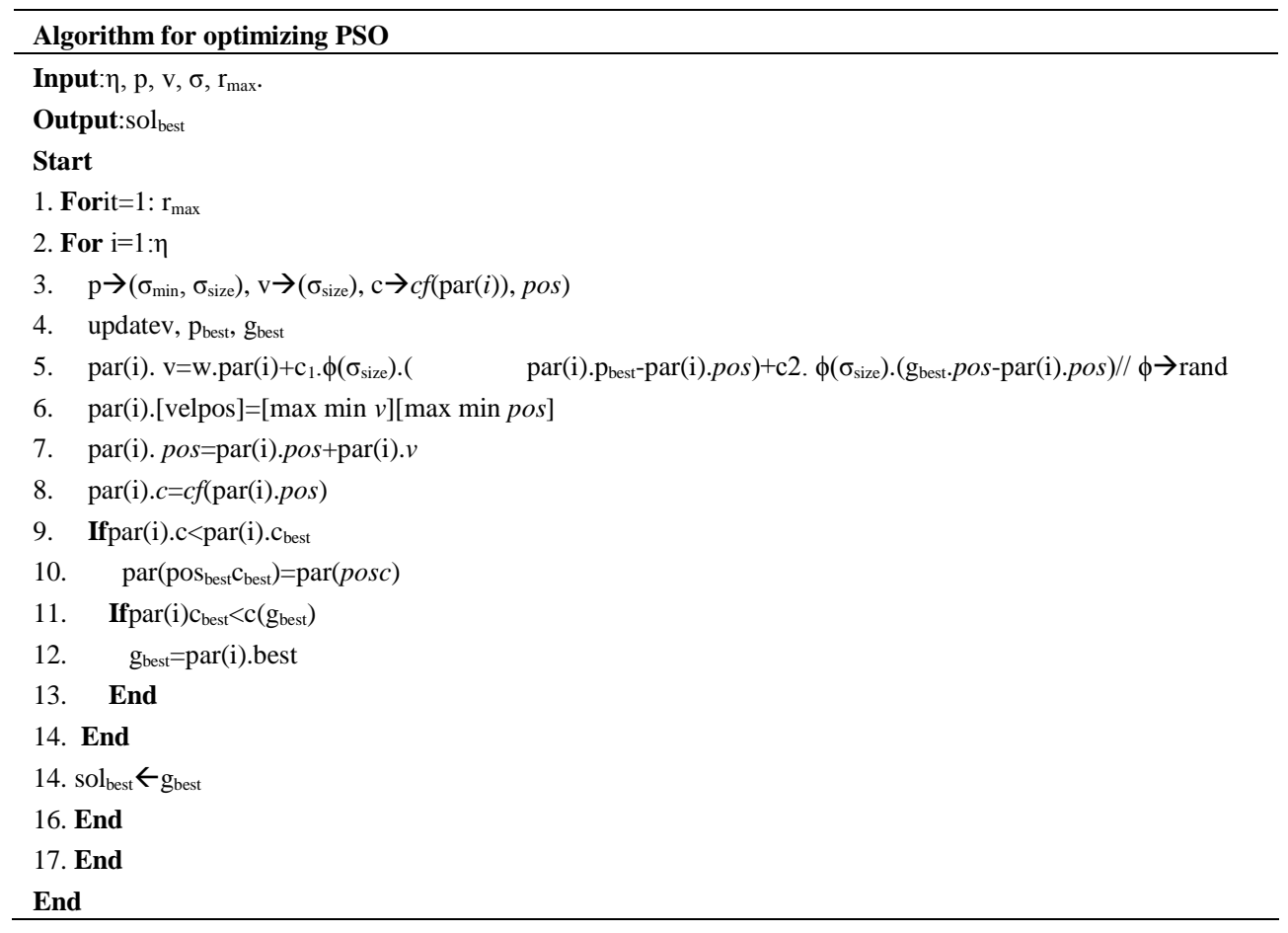

The number of clusters is equivalent to the product of the number of nodes and probability of node to opt for becoming a cluster head. The algorithm initially finds the position, velocity, and cost of the particle using minimum variance, size of variance, and position attributes (Line-3) for all number of populations $(\eta)$. It then opts for updating the velocity, personal best and global best (Line-4) using position and cost attribute of the particle. The next part of the study considers updating the other parameters for maximum iteration rounds using the empirical expression of velocity $\mathrm{v}$ (Line-5). The next part of the algorithm is for applying the maximum and minimum limits of velocity (Line-6) followed by updating the position particles using empirical expression highlighted in Line-7. The cost attribute is further evaluated considering the particle position (Line-8). If the cost of the particle is found to be less than the best value of the cost (Line-9) than the algorithm chooses to assign the normal position as best position and normal cost as best cost (Line-10). However, if the personal best cost of the particle is found to be less than the global best cost than (Line-11)

Novel Scheme for Minimal Iterative PSO Algorithm for Extending Network Lifetime of .... (Hemavathi P) 
the algorithm applies the personal best cost to the global best cost (Line-12). This global best cost is finally considered to be the best solution itself (Line-12 and Line-15). For better control of the iteration, the algorithm can restrict the iteration of best cost to any value (in the proposed system, we have iterated it to 20 times to found better outcomes). Therefore, the proposed algorithm could successfully optimize the best position of the particle which is directly mapped to the selection process of the cluster head. Hence, optimizing the existing PSO offers better control of the selection mechanism that directly effects energy conservation in the data forwarding process.

\subsection{Algorithm for Enhancing Network Lifetime}

This algorithm is responsible for improving the network lifetime of the sensor network where the focus is laid to the energy being depleted by the clusterhead itself. It is because if the transmittance energy of clusterhead $E_{t x}$ is reduced than a significant amount of the energy could be postponed for a faster rate of depletion of the battery of a node. The algorithm takes the input of $\mathrm{E}$ (residual energy) and $\mathrm{N}$ (number of nodes) that after processing leads to a generation of output as $\mathrm{E}_{\text {out }}$ (revised residual energy).

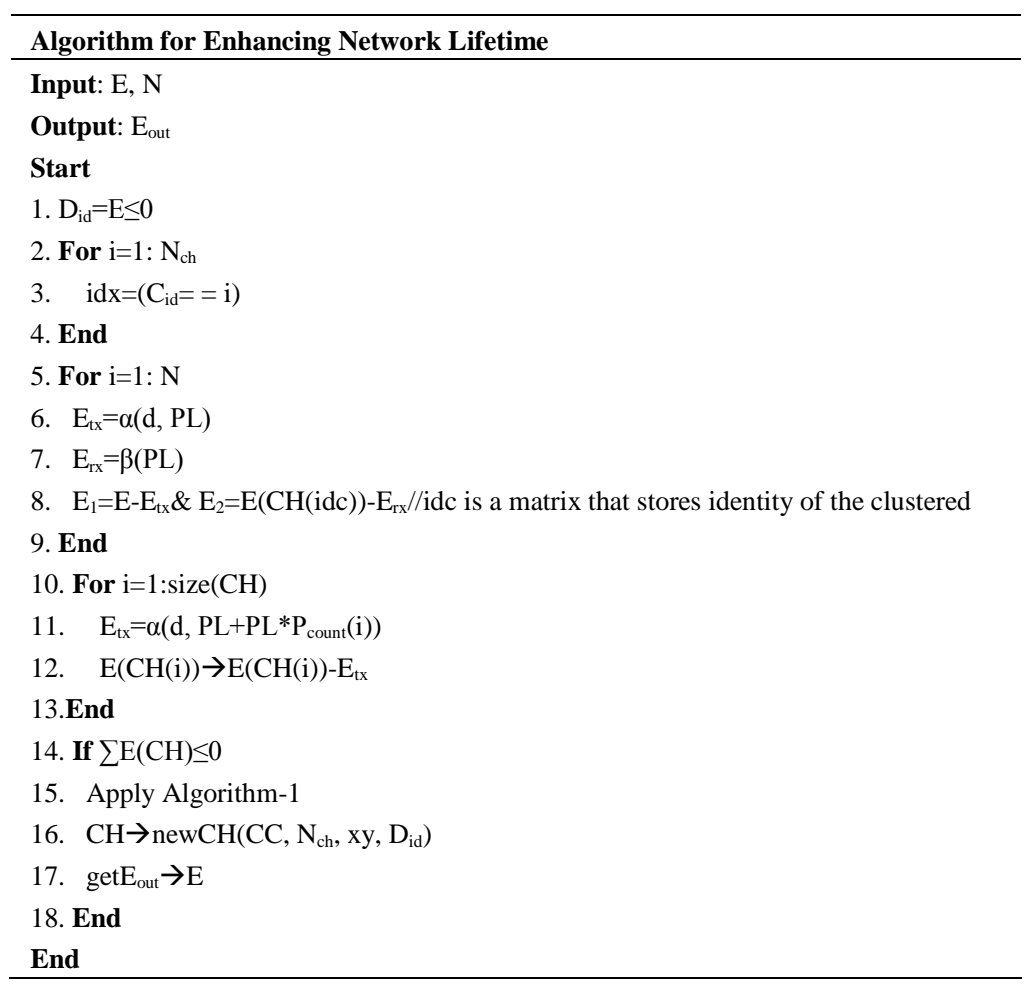

The algorithm formulates a simple condition for identifying dead node $\mathrm{D}_{\text {id }}$ as the node with lesser residual energy E (Line-1). The initial part of the study is to obtain the center point of the cluster, and then it obtains all the index of the ith cluster (Line-3) for all the values of clusterheads (Line-2). For all the number of nodes (Line-5), the algorithm applies the first order radio-energy modeling to apply function for computing transmittance energy $\alpha$ (Line-6) and receiving energy $\beta$ (Line-7). A closer look at the expression shows that transmittance energy depends on Euclidean distance $d$ and packet length PL (Line-6) while receiving energy only depends on packet length (Line-7). The next step of the algorithm is mainly used for minimizing both transmittance energy $E_{1}$ and receiving energy $E_{2}$ using the expression shown in Line- 8 . The complete process of minimization of the energy $\left(E_{1}\right.$ and $\left.E_{2}\right)$ is only from member node to clusterhead and clusterhead to another clusterhead. The algorithm now revises the computation by considering only communication from clusterhead to base station (Line-10) where first the coordinates of the cluster heads are obtained followed by computation of transmittance energy $\mathrm{E}_{\mathrm{tx}}$ (Line-11) by applying mathematical expressions of first-order radio-energy model considering the input arguments of Euclidean distance between the cluster head and base station, length of the data packet, and number of cluster head. It then reduces the energy by subtracting it with the $\mathrm{E}_{\mathrm{tx}}$ recently computed (Line-12). The process is carried out for all the active clusterheads in the sensor network. Interestingly, in case of the dead of any clusterhead, the algorithm applies proposed PSO (Line-15) to compute the new clusterhead (Line-14). The complete computation is carried out 
to find the dead node and identifying the position of the dead nodes so that simultaneous updates can be carried on the PSO that further optimizes the operation process involves in proposed PSO algorithm. In this case, the proposed PSO algorithm is applied considering the input arguments of (a) best position CC, (b) number of clusterheads $\mathrm{N}_{\mathrm{ch}}$, (c) position information by, and (d) dead node identity $\mathrm{D}_{\text {id }}$. Therefore, irrespective of any position of the sensor node, the proposed algorithm is meant for reducing the transmittance energy of the cluster head. Hence, the network lifetime of the wireless sensor network is significantly enhanced with lesser dependencies on the position of the sensor nodes considering both dense and scarce network. This indirectly minimizes the computational complexities associated with the implementation of swarm intelligence algorithm in a wireless sensor network. The next section discusses the results obtained by implementing the proposed algorithm.

\section{RESULT ANALYSIS}

The implementation of the proposed algorithm is carried out using the Matlab and assessed concerning both energy and data delivery parameters for assessing the effect of proposed PSO-based optimization. The study outcome was compared with conventional LEACH algorithm for 1800 simulation rounds. The outcomes show that proposed system with PSO offers better network lifetime (Figure 2) and better throughput (Figure 3) as compared to LEACH.

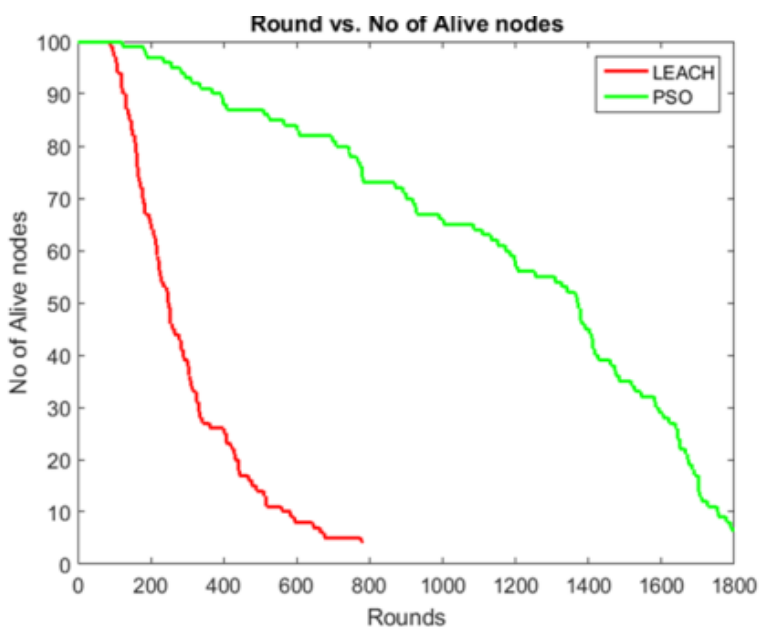

Figure 2. Comparative Analysis of Number of Alive Nodes

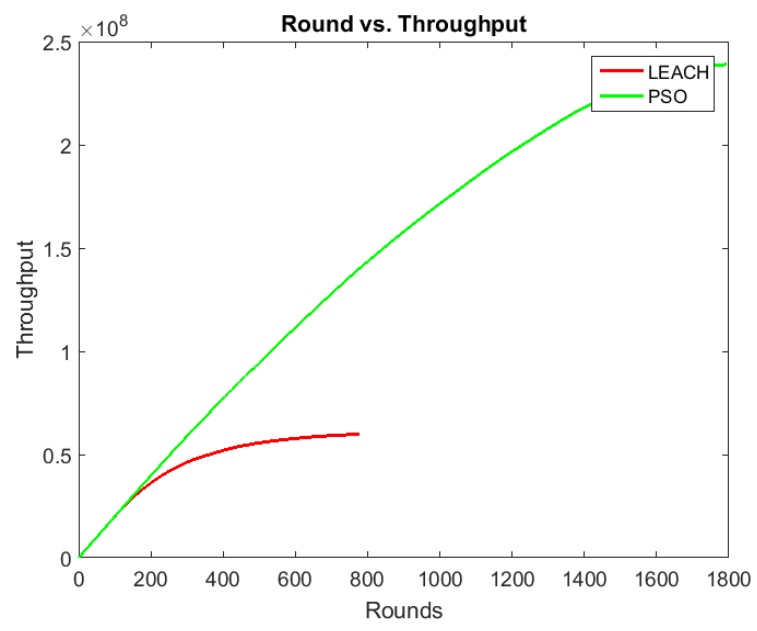

Figure 3. Comparative Analysis of Throughput

The outcome shows that proposed system offers significantly increased retention of alive nodes with $50 \%$ improvement as considered too conventional LEACH algorithm whereas it also ensures a smooth gradient ascent for throughput curve showing better predictability of the throughput performance whereas the curve of LEACH witness $100 \%$ of node death just before even completing $50 \%$ of the simulation iteration. At the same time, proposed system doesn't have any form of storage dependencies, and hence its computational complexity is highly negligible.

\section{CONCLUSION}

At present, there are various research-based techniques focused on implementing PSO for solving multiple problems in a wireless sensor network that comes under the ranges of clustering, energy conservation, and localization. Although existing PSO-based techniques have made some good problems in obtaining the better outcome, we find that such solutions have overlooked the problems associated with the computational complexity in PSO due to its higher involvement of iteration. The proposed system proved that it is quite feasible to control the iteration of PSO and still obtain better energy efficiency and enhanced data delivery performance in a wireless sensor network in comparison to the existing system. 


\section{REFERENCES}

[1] W. Sun, Z. Yang, X. Zhang, and Y. Liu, "Energy-Efficient Neighbor Discovery in Mobile Ad Hoc and Wireless Sensor Networks: A Survey," in IEEE Communications Surveys \& Tutorials, vol. 16, no. 3, pp. 1448-1459, Third Quarter 2014.

[2] Y. Gu, F. Ren, Y. Ji and J. Li, "The Evolution of Sink Mobility Management in Wireless Sensor Networks: A Survey," in IEEE Communications Surveys \& Tutorials, vol. 18, no. 1, pp. 507-524, First quarter 2016

[3] K. A. Shim, "A Survey of Public-Key Cryptographic Primitives in Wireless Sensor Networks," in IEEE Communications Surveys \& Tutorials, vol. 18, no. 1, pp. 577-601, First quarter 2016.

[4] M. Z. Hasan; H. Al-Rizzo; F. Al-Turjman, "A Survey on Multipath Routing Protocols for QoS Assurances in RealTime Wireless Multimedia Sensor Networks," in IEEE Communications Surveys \& Tutorials, vol.PP, no.99, pp.1-1

[5] S. R. Jondhale, R. S. Deshpande, S. M. Walke, and A. S. Jondhale, "Issues and challenges in RSSI based target localization and tracking in wireless sensor networks," 2016 International Conference on Automatic Control and Dynamic Optimization Techniques (ICACDOT), Pune, 2016, pp. 594-598.

[6] Y. M. Yusof, A. K. M. M. Islam and S. Baharun, "An experimental study of WSN transmission power optimisation using MICAz motes," 2015 International Conference on Advances in Electrical Engineering (ICAEE), Dhaka, 2015, pp. $182-185$

[7] SatchidanandaDehuri, "Integration of Swarm Intelligence and Artificial Neural Network," World Scientific, 2011

[8] Parsopoulos, Konstantinos E, Particle Swarm Optimization, and Intelligence: Advances and Applications: Advances and Applications, IGI Global, 2010

[9] Maurice Clerc, Particle Swarm Optimization, John Wiley \& Sons, 2013

[10] Hemawathi.p and T G Basavaraju, "An Investigational Study of Energy Conservation Techniques in Hierarchical Routing Protocols in Wireless Sensor Network", International Journal of Computer Applications 101(7):14-19, September 2014.

[11] Y. Zhou, N. Wang, and W. Xiang, "Clustering Hierarchy Protocol in Wireless Sensor Networks Using an Improved PSO Algorithm," in IEEE Access, vol. 5, no., pp. 2241-2253, 2017.

[12] Q. Ni, Q. Pan, H. Du, C. Cao, and Y. Zhai, "A Novel Cluster Head Selection Algorithm Based on Fuzzy Clustering and Particle Swarm Optimization," in IEEE/ACM Transactions on Computational Biology and Bioinformatics, vol. 14, no. 1, pp. 76-84, Jan.-Feb.

[13] W. Xiang, N. Wang, and Y. Zhou, "An Energy-Efficient Routing Algorithm for Software-Defined Wireless Sensor Networks," in IEEE Sensors Journal, vol. 16, no. 20, pp. 7393-7400, Oct.15, 2016.

[14] W. Guo, J. Li, G. Chen, Y. Niu and C. Chen, "A PSO-Optimized Real-Time Fault-Tolerant Task Allocation Algorithm in Wireless Sensor Networks," in IEEE Transactions on Parallel and Distributed Systems, vol. 26, no. 12, pp. 3236-3249, Dec. 1, 2015.

[15] J. RejinaParvin and C. Vasanthanayaki, "Particle Swarm Optimization-Based Clustering by Preventing Residual Nodes in Wireless Sensor Networks," in IEEE Sensors Journal, vol. 15, no. 8, pp. 4264-4274, Aug. 2015.

[16] T. Y. Wu and C. H. Lin, "Low-SAR Path Discovery by Particle Swarm Optimization Algorithm in Wireless Body Area Networks," in IEEE Sensors Journal, vol. 15, no. 2, pp. 928-936, Feb. 2015.

[17] J. Yang, H. Zhang, Y. Ling, C. Pan, and W. Sun, "Task Allocation for Wireless Sensor Network Using Modified Binary Particle Swarm Optimization," in IEEE Sensors Journal, vol. 14, no. 3, pp. 882-892, March 2014.

[18] M. N. Rahman and M. A. Matin, "Efficient algorithm for prolonging network lifetime of wireless sensor networks," in Tsinghua Science and Technology, vol. 16, no. 6, pp. 561-568, Dec. 2011.

[19] D. T. Ho, E. I. Grøtli, P. B. Sujit, T. A. Johansen and J. B. De Sousa, "Performance evaluation of cooperative relay and Particle Swarm Optimization path planning for UAV and wireless sensor network," 2013 IEEE Globecom Workshops (GC Wkshps), Atlanta, GA, 2013, pp. 1403-1408

[20] V. Loscri, E. Natalizio, F. Guerriero, G. Aloi, "Particle swarm optimization schemes based on consensus for wireless sensor networks," ACM Digital Library, pp. 77-84, 2012

[21] Y. C. Chen and J. R. Jiang, "Particle Swarm Optimization for Charger Deployment in Wireless Rechargeable Sensor Networks," 2016 26th International Telecommunication Networks and Applications Conference (ITNAC), Dunedin, 2016, pp. 231-236.

[22] J. Du, L. Liu, and Y. Ling, "An error beacon filtering algorithm based on particle swarm optimization for underwater wireless sensor networks," 2016 IEEE International Conference on Ubiquitous Wireless Broadband (ICUWB), Nanjing, 2016, pp. 1-4.

[23] Z. Chen, X. Li, B. Lv and M. Jia, "A Self-Adaptive Wireless Sensor Network Coverage Method for Intrusion Tolerance Based on Particle Swarm Optimization and Cuckoo Search," 2015 IEEE Trustcom/BigDataSE/ISPA, Helsinki, 2015, pp. 1298-1305.

[24] L. Zhi-Kun and L. Zhong, "Node self-localization algorithm for wireless sensor networks based on modified particle swarm optimization," The 27th Chinese Control and Decision Conference (2015 CCDC), Qingdao, 2015, pp. $5968-5971$

[25] S. Thilagavathi and B. G. Geetha, "Energy-Aware Swarm Optimization with Intercluster Search for Wireless Sensor Network," Hindawi Publishing Corporation-Scientific World Journal, 2015

[26] R. S. Elhabyan and M. C. E. Yagoub, "Particle swarm optimization protocol for clustering in wireless sensor networks: A realistic approach," Proceedings of the 2014 IEEE 15th International Conference on Information Reuse and Integration (IEEE IRI 2014), Redwood City, CA, 2014, pp. 345-350.

[27] Trong-Thua Huynh, Anh-Vu Dinh-Duc, Cong-Hung Tran and Tuan-Anh Le, "Balance Particle Swarm Optimization and gravitational search algorithm for energy efficient in heterogeneous wireless sensor networks," The 2015 IEEE 
RIVF International Conference on Computing \& Communication Technologies - Research, Innovation, and Vision for Future (RIVF), Can Tho, 2015, pp. 175-179.

[28] I. F. M. Zain and S. Y. Shin, "Distributed Localization for Wireless Sensor Networks Using Binary Particle Swarm Optimization (BPSO)," 2014 IEEE 79th Vehicular Technology Conference (VTC Spring), Seoul, 2014, pp. 1-5.

[29] C. Cao, Q. Ni and X. Yin, "Comparison of Particle Swarm Optimization algorithms in Wireless Sensor Network node localization," 2014 IEEE International Conference on Systems, Man, and Cybernetics (SMC), San Diego, CA, 2014, pp. 252-257.

[30] Z. Jing, T. Le and Z. Shuaibing, "A novel clustering algorithm based on particle swarm optimization for wireless sensor networks," The 26th Chinese Control and Decision Conference (2014 CCDC), Changsha, 2014, pp. 2769-2772.

[31] SK. Riaz, V. Srirammanoj, "Sufficient Authentication for Energy Consumption in Wireless Sensor Networks", TELKOMNIKA (Telecommunication Computing Electronics and Control) Vol. 16, No. 2, pp. $316 \sim 322$, November 2015.

[32] Enyan Sun, Chuanyun Wang, Feng Tian, "A Survey on Clustering Routing Protocols Based on PSO in WSN", TELKOMNIKA (Telecommunication Computing Electronics and Control) Vol.12, No.7, pp. $5484 \sim 5490$, July 2014.

[33] [33] Wu Rui, Xia Kewen, Bai Jianchuan, Zhang Zhiwei, "A Novel Clustering Routing Protocol in Wireless Sensor Network", TELKOMNIKA (Telecommunication Computing Electronics and Control) Vol.12, No.6, pp. $4283 \sim 4289$, June 2014

\section{BIOGRAPHIES OF AUTHORS}

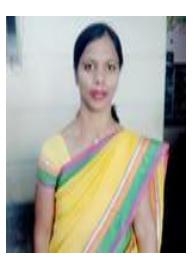

Hemavathi P obtained B.E from Manipal Institute of Technology, Manipal (University of MAHE), India. She completed M.Tech from Dr. Ambedkar Institute of Technology Bangalore, (VTU) India. Her areas of interest are Wireless Sensor Networks, Adhoc Networks. Currently, she is pursuing Ph.D. at Jain University, Bangalore.

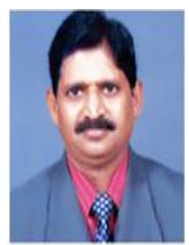

Dr. Nandakumar A N obtained his B.Sc in 1972, BE degree in 1976 both from University of Mysore, India, and Ph.D. from Berhanpur university in the year 2008 after getting M.Tech from University Of Roorkee (present IIT ROORKEE) in the year 1990. He is working as Professor, New Horizon College of Engineering in the Department of Computer science and engineering, Bangalore. His research is in the field of Image processing, pattern recognition, internet of things and others. He is a life member of ISTE. 